Musées, Patrimoine et Culture scientifiques et techniques

$187 \mid 2020$

janvier-février 2020

\title{
Questionner les liens sociétés-Terre
}

\section{Nicolas Kramar}

\section{OpenEdition}

Journals

Édition électronique

URL : http://journals.openedition.org/ocim/3522

DOI : 10.4000/ocim.3522

ISSN : 2108-646X

Éditeur

OCIM

Édition imprimée

Date de publication : 1 janvier 2020

Pagination : 14-19

ISSN : 0994-1908

Référence électronique

Nicolas Kramar, "Questionner les liens sociétés-Terre », La Lettre de I'OCIM [En ligne], 187 | 2020, mis en ligne le 01 janvier 2021, consulté le 25 janvier 2021. URL : http://journals.openedition.org/ocim/ 3522 ; DOI : https://doi.org/10.4000/ocim.3522

Ce document a été généré automatiquement le 25 janvier 2021.

Tous droits réservés 


\title{
Questionner les liens sociétés-Terre
}

\author{
Nicolas Kramar
}

Ce trophée est un hybride : ni naturel (il ne s'agit pas d'un trophée classique de collection de sciences naturelles), ni culturel, il ne s'agit pas non plus d'une œuvre d'art. Collection Anthropocène du musée de la Nature du Valais.

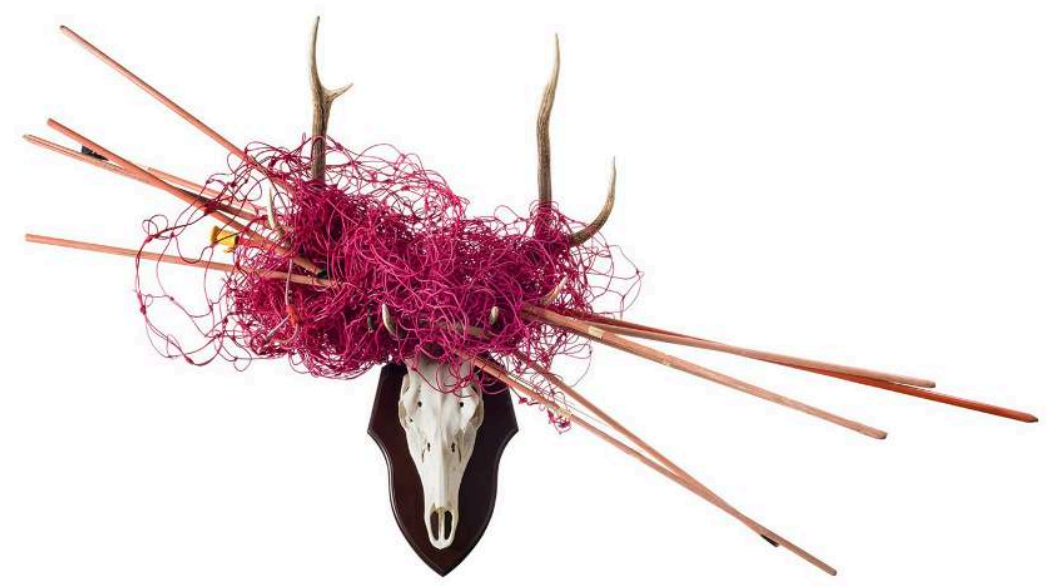

() Michel Martinez - Musées cantonaux

$1 \quad$ L'idée de vouloir inscrire dans l'histoire de la Terre l'influence croissante des activités humaines sur l'environnement date du XIX ${ }^{e}$ siècle. Cependant, il faut attendre l'année 2000 pour que cette idée ressurgisse et connaisse un réel développement. 
2 Des expositions de musées de sciences et techniques, d'art, d'histoire ou d'anthropologie ont traité de ce sujet depuis 2013. Les musées d'histoire naturelle ont été jusqu'à récemment en retrait sur ce thème. Il a fallu attendre 2016, au musée de la Nature du Valais en Suisse (après l'ouverture en 2014 d'une salle dédiée à l'Anthropocène dans son exposition permanente), et 2017, au muséum d'Histoire naturelle du Carnegie de Pittsburgh aux États-Unis, pour qu'on voie se développer de grandes expositions temporaires sur ce thème (Robin, 2018 et Oliveira et al., soumis pour publication).

3 Le traitement de ce thème dans les musées de sciences naturelles, que ce soit au sein d'expositions temporaires ou permanentes, confirme son actualité. Le concept d'Anthropocène ne peut être réduit à un effet de mode et il reste largement moins utilisé dans les médias que les notions de changement climatique, de développement durable, de crise environnementale ou, depuis peu, de biodiversité. Pourtant, ces diverses notions prises individuellement peinent à rendre compte de façon globale des enjeux environnementaux actuels et futurs. Le relatif succès de la notion d'Anthropocène réside en partie dans sa capacité à le faire. Plus encore, son pouvoir heuristique ouvre de nouvelles perspectives pour les musées de sciences naturelles.

\section{Controverse}

4 En tant que concept émergent, l'Anthropocène est l'objet de nombreux discours et d'interprétations différentes, tant du point de vue des sciences de l'environnement que de celui des sciences humaines et sociales.

5 Au sein de la communauté des géologues en charge de l'échelle des temps géologiques, la controverse est d'abord technique et concerne la définition d'un niveau stratigraphique de référence pour situer le début de l'Anthropocène. En 2009, un groupe de travail a été mis en place par la Commission internationale de stratigraphie afin d'étudier la possibilité d'insérer l'Anthropocène au sein de l'échelle des temps géologiques. Dix ans plus tard, en 2019, ce groupe a conclu qu'il était pertinent d'introduire l'Anthropocène dans l'échelle des temps géologiques et que le milieu du $\mathrm{XX}^{e}$ siècle devrait constituer sa base. Malgré la quasi-unanimité des membres de ce groupe - 29 sur 34 (Subramanian, 2019) - leurs propositions font débat au sein de la communauté géologique. S'il est évident que la méthodologie utilisée pour définir des périodes (séries) géologiques antérieures est difficilement applicable pour un processus en cours, on peut néanmoins regretter que la même rigueur à critiquer les propositions relatives à l'Anthropocène ne soit pas aussi appliquée à l'Holocène, notamment sur la très faible capacité de son niveau repère à perdurer dans le temps (car défini dans les glaces du Groenland). 
L'Anthropocène inscrit les changements globaux actuels dans l'histoire de la Terre et offre de nouvelles perspectives : elles sont symbolisées par de multiples lignes partant de l'Anthropocène et liant diverses thématiques au sein de l'exposition Objectif-Terre, vivre l'Anthropocène. Scénographie proposée par Marie Velardi.

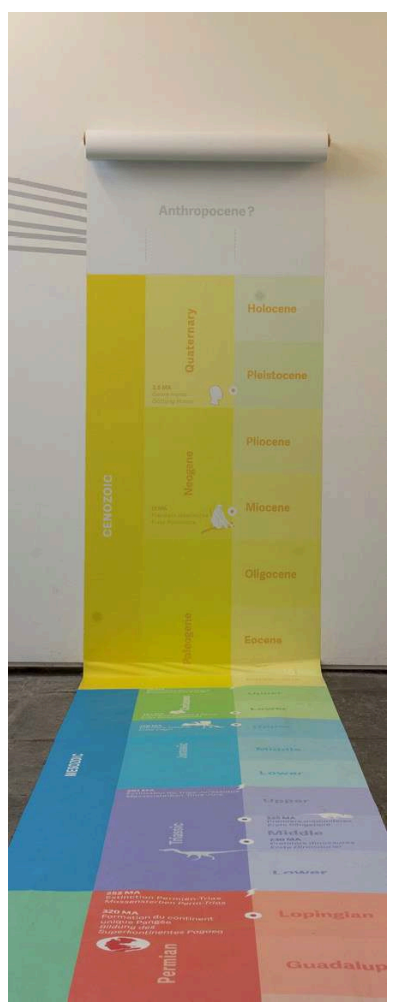

(c) Robert Hofer -Musées cantonaux

6 L'intérêt pour l'Anthropocène génère, sur le sérail réduit des géologues-stratigraphes, le sentiment d'une forme de pression sociale que Stanley Finney, membre du comité exécutif de l'Union internationale des Sciences géologiques, résume par l'impression de se trouver dans un phare avec un tsunami en approche (feel « like a lighthouse with a huge tsunami wave coming at it» $)^{1}$. Cette situation a été analysée par des historiens des sciences qui l'ont mise en parallèle avec l'émergence de la notion d'environnement durant le XX ${ }^{e}$ siècle (Warde, Robin, Sörlin, 2017). Ainsi, certains débats portent encore la marque du "grand partage» entre les sciences physiques et les autres types de discours, et relèvent d'arguments et de perspectives internalistes à l'histoire des sciences, rejetant la part du contexte sociétal dans lequel la production de savoirs scientifiques s'insère.

$7 \mathrm{Du}$ point de vue des sciences humaines et sociales, le mot "Anthropocène » est inadéquat puisque, considéré à l'échelle de l'histoire humaine, il semble renvoyer à l'idée d'une humanité unique, homogène, dans laquelle toutes les sociétés partageraient les mêmes valeurs et auraient les mêmes responsabilités. C'est ainsi que d'autres mots ont été proposés (tels que "Capitalocène", "Anglocène ", "Occidentalocène », etc.) afin de préciser les origines de la situation présente. La discussion sur les causes de l'Anthropocène a conduit à une multitude d'interprétations (Bonneuil et Fressoz, 2013) que l'on peut classer en deux grands groupes, selon qu'elles relèvent $\mathrm{du}$ bon ou du mauvais Anthropocène. Le premier se conçoit dans une perspective techno-utopiste qui fait le pari que le génie humain, grâce à ses technologies, va réparer les dégâts qu'il a causés de façon non-intentionnelle. À 
l'inverse, le mauvais Anthropocène interroge les dimensions symboliques qui fondent la relation des sociétés à leurs environnements, et donc in fine la science elle-même, pour intégrer dans le débat les mythes et récits produits par les sociétés occidentales.

Malgré des interprétations divergentes, voire contradictoires, il existe un consensus sur l'idée d'effets anthropiques profonds et ubiquistes sur le système Terre. La magnitude de ces changements est telle, ne serait-ce qu'en terme de modification de la biodiversité, qu'ils sont significatifs au regard de l'histoire de la Terre. De ce fait, il est irréfutable que l'idée d'Anthropocène relève aussi des sciences de la Terre pour qui - à l'échelle de l'histoire de la Terre, et non pas celle de l'histoire humaine - la cause de la situation présente est Homo sapiens, quels qu'en soient les sous-groupes. Ce point de vue, en dépit de ses limites, explique probablement la raison pour laquelle le mot Anthropocène perdure et qu'aucune des autres propositions mentionnées précédemment n'a pu le remplacer. On peut donc accepter qu'il soit «équivoque et partiellement inapproprié » (Bourg, 2019). Cette référence au temps géologique permet de distinguer, du point de vue des sciences de l'environnement, la notion d'Anthropocène de deux autres expressions couramment utilisées pour qualifier la situation écologique actuelle. Tout d'abord, l'Anthropocène se distingue de la notion de changement global puisqu'il intègre une dimension géologique. Ensuite, son caractère irréversible (Waters et al., 2016), par définition, rend caduc l'usage de la notion de "crise environnementale»: le terme de crise supposant un possible retour aux conditions initiales (Bourg, 2015).

\section{Heuristique de l'Anthropocène}

Les discours sur l'Anthropocène mobilisent toute une série de thèmes qui, pris individuellement, ne sont pas nouveaux (biodiversité, systémique, modernité, changement climatique, etc.) et font largement consensus. C'est la mise en lien de tous ces éléments que permet l'Anthropocène et qui aboutit à renouveler la perception des enjeux environnementaux. Il s'agit donc d'une notion transdisciplinaire à fort potentiel heuristique, offrant de nouvelles perspectives sur les questions environnementales et permettant même, du point de vue muséologique, de mettre à distance des fondements de nos pratiques professionnelles (Kramar, 2016).

10 L'Anthropocène ne se réduit à aucun des problèmes environnementaux actuels. Dans cette perspective, les problématiques environnementales ne sont pas sectorisées mais liées, intégrées dans une vision systémique qui offre une large perspective sur l'état de la Terre. Vision qui révèle aussi des problèmes moins connus tels que, entre nombreux autres, l'appauvrissement des sols ou la diminution de l'oxygène dans les mers et les océans. Pour l'illustrer, l'Anthropocène est souvent associé au concept de limites planétaires. Il s'agit d'un modèle qui vise à caractériser l'état général du système Terre en définissant et en calculant deux paramètres pour neuf thématiques environnementales de première importance : tout d'abord une limite à ne pas dépasser pour garder une forme de stabilité du système et ensuite un seuil au-delà duquel le système n'est plus du tout gérable et pourrait présenter des modifications brutales. Le modèle des limites planétaires permet de comparer l'importance relative de différents problèmes environnementaux. Plus encore, si l'on s'intéresse aux raisons à l'origine du déclin de la biodiversité - l'une des deux limites planétaires dont le seuil a été dépassé on découvre que celle-ci a comme premier facteur l'usage des sols. Autrement dit, l'état 
désastreux de la biodiversité n'a pas comme principal facteur le changement du climat (pour lequel la limite a été dépassée, mais non le seuil). Ce constat diffère des discours médiatiques et politiques dominants. Le modèle des limites planétaires avait été mis en scène dans l'exposition Objectif-Terre du musée de la Nature du Valais ${ }^{2}$ et avait ainsi permis à de nombreux visiteurs de découvrir l'étendue et la variété des problèmes environnementaux. Ce modèle est actuellement présenté dans des expositions temporaires d'autres musées de sciences naturelles, tels que le musée d'Histoire naturelle de Bern (exposition Apocalypse - une fin sans fin) ou au Museu Blau, musée des Sciences naturelles de Barcelone (exposition Som Natura).

11 L'Anthropocène inscrit le présent dans l'histoire géologique: ce qui signifie que se croisent temps géologiques et historiques. La réflexion sur les causes à l'origine de la situation actuelle nécessite de questionner les fondements anthropologiques des relations des sociétés avec leur environnement. Car on ne saurait se limiter à une perspective qui considérerait une humanité unique où ne serait en jeu que la démographie. La manière qu'ont les sociétés de faire monde est un processus contingent, en constante transformation dans le temps et l'espace. L'Anthropocène doit tout autant intégrer ce type de savoir que celui des sciences du système Terre. Les conséquences des activités humaines ne modifient de façon significative le système Terre que depuis quelques dizaines, voire centaines d'années. Cela permet de distinguer la notion d'anthropisation (notion qu'on pourrait généraliser à d'autres espèces vivantes qui, avec le temps, ont affecté les nouveaux milieux qu'elles ont colonisés) de l'Anthropocène (Descola, 2018). Il est alors nécessaire d'en comprendre les raisons anthropologiques. Les références en géographie culturelle, en anthropologie, en sociologie, en histoire et en philosophie sont nombreuses (avec des auteurs comme Berque, Bourg, Descola, Dumont, Latour) pour mettre en évidence l'apparition d'une ontologie particulière qui se développe dès la Renaissance en Occident.

Cette ontologie naît dans un mouvement d'objectivation du monde naturel et résulte dans une forme de distanciation symbolique de la société avec la nature, qui se renforcera au cours du temps. Dans ce cadre, la nature est perçue comme une machine, déterminée selon des lois mécaniques/physiques immuables, alors que la culture est comprise comme la liberté des humains d'en disposer sans contrainte. Toute la modernité - y compris la naissance de la science moderne au XVIIe siècle et par conséquent les savoirs présentés dans les musées de sciences naturelles - se fonde sur cette dichotomie. Du fait des effets en retour de l'action humaine sur les conditions de vie sur la Terre (Descola, 2018), l'Anthropocène signe les limites de ce paradigme et révèle le caractère hybride (Latour, 1991), naturel et culturel, de tout ce qui nous entoure, marquant la fin de la modernité. Cette ontologie particulière, qui naît à la Renaissance et se développe progressivement avant d'être largement exportée dans le monde entier, détermine l'essentiel des pratiques qui ont un fort impact environnemental. 
En arrivant dans lasalle de l'exposition Objectif-Terre, vivre l'Anthropocène relativeà la modernité, les multiples lignes parcourant l'exposition s'alignent pour faire apparaitre une grille de perspective. Elle représente la distanciation symbolique qui s'opère en Occident dès la Renaissance. Divers automates (en particulier provenant de l'atelier de François Junod, automatier) peuplaient ce monde conçu comme une machine. Scénographiede Marie Velardi.

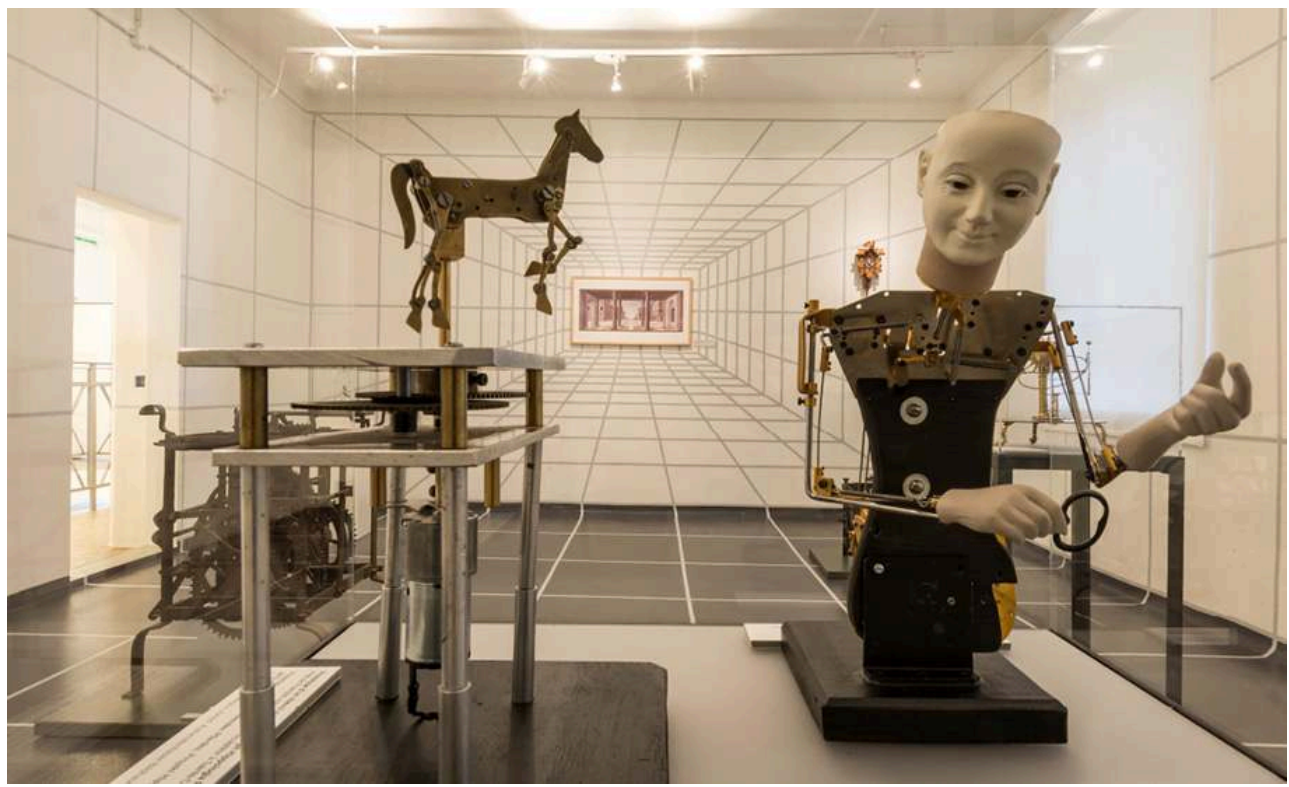

(c) Robert Hofer -Musées cantonaux

Penser l'Anthropocène, c'est ainsi l'occasion de mettre à distance les mythes et récits qui fondent nos sociétés occidentales en questionnant certaines évidences. Le musée de la Nature du Valais a intégré à sa collection un trophée d'un cerf qui était mort d'épuisement après que ses bois se soient accrochés à des piquets et filets de ski. Il en résulte un trophée qui n'est ni une œuvre d'art (quand bien même on pourrait le suspecter), ni un trophée classique que l'on retrouve dans toutes les collections de musée de sciences naturelles. Ni culturel, ni naturel, il est le parfait exemple d'un objet hybride de notre époque.

L'Anthropocène pointe les limites des grandes dichotomies modernes, au premier chef la séparation entre nature et culture. Pour penser leur imbrication, il a recours notamment à la notion de système, qui est au cœur des savoirs issus des sciences du système Terre. La dimension systémique s'impose comme un outil de pensée alternatif au paradigme moderne, permettant ainsi d'aller au-delà des notions couramment mobilisées pour traiter des enjeux environnementaux. Par exemple, la perspective la plus courante du développement durable ne remet pas en question l'idée qu'il soit possible de croître indéfiniment dans un monde fini, témoignant ainsi des limites du paradigme moderne sur lequel elle se fonde.

\section{Conclusion}

15 Au-delà des controverses inhérentes à un concept émergent, l'Anthropocène est une notion avec un fort potentiel heuristique. Elle offre un regard distancié sur l'ensemble des problèmes environnementaux en évitant l'écueil de se focaliser sur un seul problème ou de n'envisager les autres qu'au travers du changement climatique. Sur le fond, par la nécessaire intégration des savoirs qu'il entrâne, celle des sciences du 
système Terre et des sciences humaines et sociales, l'Anthropocène nous permet d'entrevoir les limites des mythes et récits de la pensée moderne. Sans bien évidemment prétendre lui fournir une alternative complète, l'Anthropocène met en valeur un concept essentiel qui entre en jeu au travers de tous les savoirs académiques qu'il mobilise et de la systémique.

Utiliser un concept avec un tel potentiel heuristique procède d'une démarche fondamentale pour les musées. Il relève d'un enjeu épistémologique consistant à intégrer dans sa communication le caractère sans cesse évoluant des savoirs et qui ne limite pas le musée à une fonction de transmetteur de connaissances scientifiques institutionnalisées. Il s'agit alors d'endosser un rôle plus philosophique et critique, interrogeant les évidences et ouvrant de nouvelles perspectives. Somme toute, rien d'autre de ce que l'on peut attendre d'une institution culturelle.

Traitement d'un gravier au glyphosate dans le village de Harie Shozu (Japon), célèbre pourses kabatas, systèmes traditionnels de gestion de l'eau douce au moyen de carpes. S'il existe de nombreuses manières de concevoir la relation au monde,les pratiques issues de la société occidentalese sont largement répandues à traversle globe.

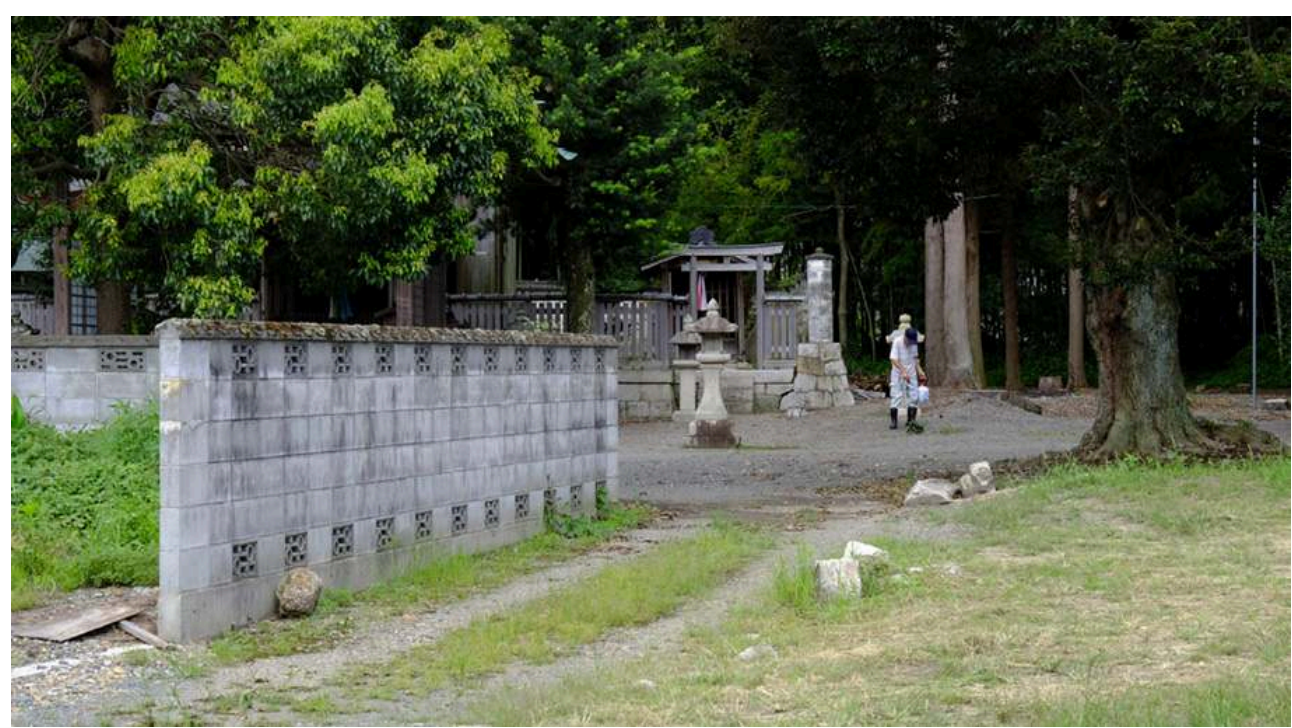

(C) Nicolas Kramar

\section{BIBLIOGRAPHIE}

Bonneuil C., Fressoz J.-B. L'événement Anthropocène. La terre, l'histoire et nous. Paris : Le Seuil, 2013, $304 \mathrm{p}$.

Bourg D. Les mots et les maux de l'environnement, Communications, vol. 96, 2015, pp. 137-144.

Bourg D. Préface du webdocumentaire Objectif-Terre, 2015, consultable sur : www.objectifterre.world 
Descola P. Humain, trop humain? in Beau R. et Larrère, C. Penser l'Anthropocène, Paris : Presses de Sciences Po, 2018, pp. 19-35.

Kramar N. Breaking the borders between nature and culture: Anthropocene as an opportunity to enrich the collections and the exhibitions, in: Conference, Icom Annual meeting, Milano, juillet 2016.

Latour B. Nous n'avons jamais été modernes. Essai d'anthropologie symétrique, Paris : La Découverte, 1991, $157 \mathrm{p}$.

Oliveira G., Dorfman É., Kramar N., Mendenhall C. D., Heller N. E., The Anthropocene in natural history museums: a productive lens of engagement, soumis pour publication.

Robin L., Boyden S. Telling the Bionarrative: A Museum of Environmental Ideas, Historical Records of Australian Science, vol. 29, n², 2018, pp. 138-152.

Subramanian M., Humans versus Earth: the quest to define the Anthropocene, Nature, vol. 572, 2019, pp. 168-170.

Warde P., Robin L., Sörlin S. Stratigraphy for the Renaissance : questions of expertise for « the environment and the Anthropocene ", The Anthropocene Review, vol. 4, n³, 2017, pp. 246-258.

Waters C. et al., The Anthropocene is functionally and stratigraphically distinct from the Holocene, Science, vol. 351, n6269, juillet 2016.

\section{NOTES}

1. Voosen, P. Atomic bombs and oil addicition herald earth's new epoch: The Anthropocene. Science News, août 2016.

2. L'exposition Objectif-Terre, vivre l'Anthropocène était présentée entre 2016 et 2017 au musée de la Nature du Valais. La scénographie était proposée par Marie Velardi.

\section{RÉSUMÉS}

Pour appréhender l'Anthropocène, il est nécessaire de dépasser certaines évidences et tenter de comprendre le système Terre dans sa globalité. Plutôt que de se limiter à certaines problématiques récurrentes mises en avant par les médias, il est fondamental que le musée et les institutions culturelles puissent à la fois faire le lien avec les connaissances scientifiques et proposer une approche critique voire philosophique du concept d'Anthropocène.

\section{INDEX}

Mots-clés : développement durable, science et société 
AUTEUR

NICOLAS KRAMAR

Directeur du musée de la Nature du Valais à Sion, en Suisse

nicolas.kramar@admin.vs.ch 